\title{
A PROPERTY OF SOLUTIONS OF ELLIPTIC DIFFERENTIAL EQUATIONS WITH DISCONTINUOUS NONLINEARITIES
}

\author{
C. A. STUART AND J. F. TOLAND
}

\section{Introduction}

Let $\Omega$ be a bounded domain in $\mathbb{R}^{n}$ which has a boundary $\partial \Omega$ of class $C^{2}$ if $n \geqslant 2$ or an open interval when $n=1$. We shall follow the notation of [1] for spaces of realvalued functions on $\Omega$.

Let $L$ denote the linear, second order uniformly elliptic operator (see [1] for definition) which is formally defined by

$$
L u(x)=-\sum_{i, j=1}^{n} a_{i j}(x) \frac{\partial^{2} u(x)}{\partial x_{i} \partial x_{j}}+\sum_{i=1}^{n} a_{i}(x) \frac{\partial u(x)}{\partial x_{i}}+a(x) u(x)
$$

where $a_{i j} \in C^{0,1}(\bar{\Omega})$ and $a_{i j}=a_{j i}, a_{i} \in L^{\infty}(\Omega)(1 \leqslant i, j \leqslant n)$ and $a \in L^{\infty}(\Omega)$.

Let $\mathrm{BN}$ denote the set of all functions $f: \mathbb{R} \rightarrow \mathbb{R}$ which have bounded variation on compact intervals and which have the property that $f(t)$ lies between $f(t+)$ and $f(t-)$ for all $t \in \mathbb{R}$. The functions in $\mathrm{BN}$ need not be continuous but they can only suffer points of jump discontinuity. It is convenient to introduce the following notation. If $f \in \mathrm{BN}$,

$$
\begin{aligned}
& U(f)=\{t \in \mathbb{R}: f(t-)<f(t+)\}, \\
& D(f)=\{t \in \mathbb{R}: f(t+)<f(t-)\} .
\end{aligned}
$$

In this paper we are concerned with solutions $u$ of the Dirichlet problem

$$
\left.\begin{array}{cc}
L u(x)=f(u(x))+p(x), & x \in \Omega \\
u(x)=0, & x \in \partial \Omega
\end{array}\right\}
$$

when $p \in L(\Omega), f \in \mathrm{BN}$. However as in earlier work $[2,3,4]$ we shall replace $(1.1)$ by the less specific problem (1.2) below for which an adequate existence theory obtains:

$$
\left.\begin{array}{l}
L u(x) \in \widetilde{f}(u(x))+p(x) \text { a.e. in } \Omega \\
u \in W^{2, r}(\Omega) \cap W_{0}^{1,2}(\Omega) .
\end{array}\right\}
$$

Here

$$
\tilde{f}(t)= \begin{cases}\{f(t)\} & \text { if } t \notin D(f) \\ {[f(t+), f(t-)]} & \text { if } t \in D(f)\end{cases}
$$

Received 5 February, 1979.

[J. LONDON MATH. SOC. (2), 21 (1980), 329-335] 
We will only consider the case where $r \geqslant 2$. Let us recall that $W^{2, n}(\Omega) \subseteq C^{0}(\bar{\Omega})$ if $r>n / 2$ and that in this case.

$$
W^{2, r}(\Omega) \cap W_{0}^{1, r}(\Omega)=\left\{u \in W^{2, r}(\Omega): u(x)=0 \text { for } x \in \partial \Omega\right\} .
$$

This paper is a sequel to [3] where we used a variational approach to study (1.2) in the case where $L=-\Delta$ and $r=2$. We showed that by assuming certain growth restrictions on $f$ it is possible to prove the existence of a solution $u^{*}$ of $(1.2)$ which is in $W^{2,2}(\Omega) \cap W_{0}^{1,2}(\Omega) \cap L^{\infty}(\Omega)$ and which minimises the extended-real-valued functional associated with (1.1), (1.2), namely:

$$
\mathscr{J}(u)=\int_{\Omega}\left\{\frac{1}{2}|\nabla u(x)|^{2}-F(u(x))-p(x) u(x)\right\} d x
$$

where $F$ is a primitive of $f$ (see [3] for further details).

This minimiser $u^{*}$ was seen to have the rather unexpected property that

$$
m\left\{x \in \Omega: u^{*}(x) \in U(f)\right\}=0
$$

where $m$ denotes $n$-dimensional Lebesgue measure.

In this paper we are specifically concerned with elaborating circumstances under which we can assert that a solution of (1.2) enjoys property $\left({ }^{*}\right)$. In Section 2 we give an example which shows that $\left(^{*}\right)$ is not a general property of solutions of (1.2).

The existence question for (1.2) has been solved using the theory of increasing maps in an ordered Banach space $[2,4]$ and we may ask whether this method invariably yields solutions which satisfy $(*)$. In $[2,4]$ it is shown that problem $(1.2)$ has a greatest and at least solution in $[\phi, \psi]=\left\{u \in W^{2, r}(\Omega): \phi \leqslant u \leqslant \psi\right\}$ provided that $\psi$ and $\phi \in W^{2, r}(\Omega)$ are super- and sub-solutions respectively, and $r>n / 2$.

In general it is not true that these greatest and least solutions enjoy $\left({ }^{*}\right)$, but nonetheless we shall show that only in exceptional (and in a sense degenerate) circumstances do they fail to have this property. As a consequence it will follow that in many cases the existence of a solution failing to satisfy $\left({ }^{*}\right)$ ensures the existence of several other solutions which do have property $\left({ }^{*}\right)$.

We illustrate this idea in Section 3 with the following special case:

$$
\begin{gathered}
-u^{\prime \prime}(x) \in f(u(x)), \quad 0<x<1, \\
u(0)=u(1)=0
\end{gathered}
$$

where $f \in \mathbf{B N}$. Under quite general conditions on $f$ we will show that (1.3) has an infinite number of distinct solutions each of which enjoys property $\left({ }^{*}\right)$.

\section{Solutions having property $\left({ }^{*}\right)$}

Throughout this section we suppose that $f \in \mathrm{BN}$ and that $p \in L(\Omega)$ for some $r>n / 2$. An element $\phi \in W^{2, r}(\Omega)$ is called a sub-solution of (1.2) if

$$
\begin{array}{crl}
L \phi(x) & \leqslant \max \{t: t \in \mathcal{f}(\phi(x))\}+p(x) & \text { a.e. on } \Omega, \\
\phi(x) \leqslant 0 & \text { on } \partial \Omega,
\end{array}
$$


and $\psi \in W^{2, r}(\Omega)$ is called a super-solution of $(1.2)$ if

$$
\begin{array}{cr}
L \psi(x) \geqslant \min \{t: t \in f(\psi(x))\}+p(x) & \text { a.e. on } \Omega \\
\psi(x) \geqslant 0 & \text { on } \partial \Omega .
\end{array}
$$

Clearly any solution of (1.2) is both a sub- and a super-solution of (1.2). In [4] the following result is established.

THEOREM 2.1. Suppose that (1.2) has a sub-solution $\phi$ and a super-solution $\psi$ in $W^{2, r}(\Omega)$ such that $\phi \leqslant \psi$ on $\bar{\Omega}$. Then, in the order interval $[\phi, \psi]=\left\{u \in W^{2, r}(\Omega): \phi \leqslant u \leqslant \psi\right\}$, the problem (1.2) has a greatest solution $\bar{u}$ and a least solution $\underline{u}$.

In the following example it is clear that such greatest and least solutions $\bar{u}, \underline{u}$, need not have property $\left({ }^{*}\right)$.

Example. Consider the special case of (1.2) with $L=-\Delta, p \equiv 0$ and $f=f_{\varepsilon}$ where

If $\varepsilon \in[-1,1]$, then $f_{\varepsilon} \in \mathrm{BN}$.

$$
f_{\varepsilon}(t)=\left\{\begin{array}{rll}
-1 & \text { if } & t<0 \\
\varepsilon & \text { if } & t=0 \\
+1 & \text { if } & t>0
\end{array}\right.
$$

By the arguments of $[2,3,4]$ there exist solutions of $(1.2)$ and by [3] at least one which has property $\left({ }^{*}\right)$. Moreover it is easy to see that for all $\varepsilon \in[-1,1]$ there exist two solutions $u$ and $\bar{u}$ such that $u(x)<0<\bar{u}(x)$ for all $x \in \Omega$. (Let $\bar{u}$ be the unique solution of the problem $-\Delta u(x)=\overline{1}$ in $\Omega, u=0$ on $\partial \Omega$, and let $u=-\bar{u}$. The maximum principle says that $\bar{u}>0>u$ on $\Omega$ and so $u$ and $\bar{u}$ solve (1.2) for any $\varepsilon \in[-1,1]$.)

However in studying the qualitative features of solutions of (1.2) we must distinguish two cases.

I. $\varepsilon=0$. If $\varepsilon=0$, then $u \equiv 0$ is a solution of (1.2) which does not satisfy $\left(^{*}\right)$. If $\phi=\psi \equiv 0$ are respectively sub- and super-solutions, then $u=\bar{u} \equiv 0$ are respectively the greatest and least solutions of $(1.2)$ in $[\phi, \psi]$ in this case.

II. $\varepsilon \neq 0$. If $\varepsilon \neq 0$, then every solution of (1.2) has property $\left({ }^{*}\right)$. To see this we simply note that $\Delta u(x)=0$ a.e. on $\{x \in \Omega: u(x)=0\}$ if $u \in W^{2, r}(\Omega)$ (Lemma 7.7 of [1]).

It is clear that this example displays many features which are typical of the more general situation, and we are lead to make the following definition.

A function $f_{1} \in \mathrm{BN}$ is called a selection of $f \in \mathrm{BN}$ if

$$
f_{1}(t) \begin{cases}=f(t) & \text { for all } t \notin U(f), \\ \in[f(t-), f(t+)] & \text { for } t \in U(f) .\end{cases}
$$

Clearly $\tilde{f}_{1}(t)=\tilde{f}(t)$ for $t \notin U(f)$. 
A function $f \in \mathrm{BN}$ is said to be non-critical with respect to $p \in L^{2}(\Omega)$ if

$$
m\{x \in \Omega: a(x) t=f(t)+p(x)\}=0
$$

for all $t \in U(f)$. (Recall that $a$ is the lowest order coefficient in the expression for $L$.)

The significance of these definitions lies in the next result.

Lemma 2.2. Suppose that $f \in \mathrm{BN}$ is non-critical with respect to $p \in L(\Omega)$. Then every solution of (1.2) has property $(*)$.

Proof. Let $u$ be a solution of (1.2). Then for each $t \in \mathbb{R}, \frac{\partial u}{\partial x_{i}}$ and $\frac{\partial^{2} u}{\partial x_{i} \partial x_{j}}=0$ a.e. on $\{x \in \Omega: u(x)=t\}$, for $1 \leqslant i, j \leqslant n,[1 ;$ Lemma 7.7]. Hence

$$
a(x) t=f(t)+p(x) \text { a.e. on }\{x \in \Omega: u(x)=t\}
$$

Since $f$ is non-critical with respect to $p, \quad m\{x \in \Omega: u(x)=t\}=0$ if $t \in U(f)$, and since $U(f)$ is denumerable,

$$
m\{x \in \Omega: u(x) \in U(f)\}=0
$$

This completes the proof of the lemma.

THEOREM 2.3. Let the hypotheses of Theorem 2.1 hold.

(a) Suppose that $f$ has a selection $f_{1}$ such that

(1) $f_{1}$ is non-critical with respect to $p$,

(2) $f_{1}(t) \geqslant f(t)$ for all $t \in U(f)$,

(3) $\psi$ is a super-solution of (1.2) for $f_{1}$.

Then $\bar{u}$ (of Theorem 2.1) has property $\left({ }^{*}\right)$ (and consequently is a solution of (1.2) for any selection of $f)$. Furthermore in $[\phi, \psi], \quad \bar{u}$ is the greatest solution of (1.2) for any selection of $f$ which satisfies (1), (2), (3) above.

(b) Suppose that $f$ has a selection $f_{2}$ such that

(4) $f_{2}$ is non-critical with respect to $p$,

(5) $f_{2}(t) \leqslant f(t)$ for all $t \in U(f)$,

(6) $\phi$ is a sub-solution of (1.2) for $f_{2}$.

Then $u$ has property $\left({ }^{*}\right)$ and in $[\phi, \psi]$ is the least solution of (1.2) for any selection which satisfies (4), (5), (6) above.

Remark 1. A sub-(super-) solution $\phi(\psi)$ of (1.2) for any selection of $f$ is a sub(super-) solution of (1.2) for any other selection of $f$ provided $\phi(\psi)$ satisfies $\left({ }^{*}\right)$. In applications this is often the case and (3) and (6) do not restrict the applicability of this result.

Remark 2. The question of whether a selection of $f$ is critical or not is easily resolved in the case where $p$ and $a$ are both constant functions on $\Omega$. Then $f$ has a non- 
critical selection $f_{1}\left(f_{2}\right)$ with $f_{1} \geqslant f\left(f_{2} \leqslant f\right)$ provided that when $t \in U(f)$ and $f(t)=a t-p$ then

$$
a t-p<f(t+) \quad(a t-p>f(t-)) .
$$

Proof of Theorem 2.3. We need only prove part (a), since the proof of (b) is similar.

Clearly $\bar{u}$ (of Theorem 2.1) is a sub-solution for $f_{1}$, and so, by Theorem 2.1 there exists in $[\bar{u}, \psi]$ a greatest solution $\bar{v}$ and a least solution $v$ for (1.2) with $f_{1}$. By the assumption that $f_{1}$ is non-critical with respect to $p$ it follows from Lemma 2.2 that both $v$ and $\bar{v}$ satisfy $(*)$. Hence $v$ and $\bar{v}$ solve (1.2) for $f$. But in $[\phi, \psi], \quad \bar{u}$ is the greatest solution of (1.2) with $f$ and $\psi \geqslant \bar{v} \geqslant v \geqslant \bar{u} \geqslant \phi$. Therefore $v=\bar{v}=\bar{u}$, and hence $\bar{u}$ satisfies $\left({ }^{*}\right)$ and is the greatest solution of $(1.2)$ for $f_{1}$ in $[\phi, \bar{\psi}]$.

As a consequence, if $f$ has a non-critical selection for $p$, then one can infer a nonuniqueness result from a priori knowledge of the existence of a solution for which $\left({ }^{*}\right)$ fails to hold.

In the next section we examine the implications of this observation in the case of an autonomous ordinary differential equation where its consequences are most striking.

\section{An autonomous ordinary differential equation}

We consider a one-dimensional version of (1.2), namely

$$
\left.\begin{array}{l}
-u^{\prime \prime}(x) \in \widetilde{f}(u(x)) \text { a.e. on }[0,1], \\
u(0)=u(1)=0, \quad u \in W^{2,2}([0,1]),
\end{array}\right\}
$$

where $f \in \mathrm{BN}$. Since all solutions of (3.1) are continuously differentiable on $[0,1]$ and $f$ is bounded on bounded sets, all solutions are in $W^{2, \infty}([0,1])$. Our main result is that if $f$ is odd and $0 \in U(f)$, then (3.1) may have an infinite number of distinct solutions.

THEOREM 3.1. Let $f \in \mathrm{BN}$ be such that

(i) $0 \in U(f)$,

(ii) $\tilde{f}(t)=-\tilde{f}(-t)$ for all $t \in \mathbb{R}$, and

(iii) either

(a) $f(t+)>0$ for all $t \in U(f)$ such that $f(t)=0$, and (3.1) has a super-solution $\psi>0$ which satisfies $\left({ }^{*}\right)$, or

(b) $f(t-)<0$ for all $t \in U(f)$ such that $f(t)=0$ and (3.1) has a sub-solution $\phi<0$ which satisfies $\left({ }^{*}\right)$.

Then (3.1) has an infinite number of distinct solutions, each of which has property $\left({ }^{*}\right)$.

Corollary. If $f \in \mathrm{BN}$ is odd, $0 \in U(f)$ and $\lim \sup (f(t) / t)<\pi^{2}$ then (3.1) has an infinite number of distinct solutions with property $\left(^{t}\right)$ if $f(t+)>0$ for all $t \in U(f)$ such that $f(t)=0$ (or if $f(t-)<0$ for all $t \in U(f)$ such that $f(t)=0$ ). 
Proof of Theorem 3.1. Suppose that (i), (ii), and (iii)(a) hold. Since $0 \in U(f), \quad f(0)=\{f(0)\}$, and so $f(0)=0$ (by (ii)). Hence $u \equiv 0$ is a solution of (3.1), which does not have property $(*)$.

By Theorem 2.3 the greatest solution $\bar{u}$ of $(3.1)$ in $[0, \psi]$ has property $\left({ }^{*}\right)$.

Let $\psi_{2}(x)=\bar{u}(x)$ for $x \in\left[0, \frac{1}{2}\right]$. Then $\psi_{2}$ is a super-solution for the problem:

$$
\left.\begin{array}{c}
-u^{\prime \prime}(x) \in \tilde{f}(u(x)) \text { a.e. on }\left[0, \frac{1}{2}\right], \\
u(0)=u\left(\frac{1}{2}\right)=0 .
\end{array}\right\}
$$

Since $\psi_{2}$ has property $(*)$, the greatest solution $\bar{u}_{2}$ of problem $(3.2)$ in $\left[0, \psi_{2}\right]$ has property $\left({ }^{*}\right)$ (by Theorem 2.3). Let

$$
w_{2}(x)= \begin{cases}u_{2}(x) & \text { if } 0 \leqslant x \leqslant \frac{1}{2} \\ -\bar{u}_{2}(1-x) & \text { if } \frac{1}{2} \leqslant x \leqslant 1 .\end{cases}
$$

Then $w_{2} \in C^{1}([0,1])$ and $w_{2}(0)=w_{2}\left(\frac{1}{2}\right)=w_{2}(1)=0$.

Furthermore, for any $h \in C_{0}^{\infty}([0,1])$,

$$
\begin{aligned}
\int_{0}^{1} w_{2}^{\prime}(x) h^{\prime}(x) d x & =\int_{0}^{\frac{1}{2}} \bar{u}_{2}(x) h^{\prime}(x) d x+\int_{\frac{1}{2}}^{1} \bar{u}_{2}^{\prime}(1-x) h^{\prime}(x) d x \\
& =-\int_{0}^{\frac{1}{2}} \bar{u}_{2}^{\prime \prime}(x) h(x) d x+\int_{\frac{1}{2}}^{1} \bar{u}_{2}^{\prime \prime}(1-x) h(x) d x
\end{aligned}
$$

Thus $w_{2}^{\prime \prime} \in L^{\infty}([0,1])$ and

$$
\begin{gathered}
-w_{2}^{\prime \prime}(x)=-\bar{u}_{2}^{\prime \prime} \in f\left(\bar{u}_{2}(x)\right)=\tilde{f}\left(w_{2}(x)\right) \text { if } 0 \leqslant x \leqslant \frac{1}{2}, \\
-w_{2}^{\prime \prime}(x)=\bar{u}_{2}^{\prime \prime}(1-x) \epsilon-\bar{f}\left(\bar{u}_{2}(1-x)\right)=\widetilde{f}\left(w_{2}(x)\right) \text { if } \frac{1}{2} \leqslant x \leqslant 1 .
\end{gathered}
$$

Thus we see that $w_{2}$ is a solution of (3.1) which enjoys property $\left({ }^{*}\right)$. Furthermore $w_{2} \neq \bar{u}$ since $w_{2}<0$ on $\left[\frac{1}{2}, 1\right]$. We can now use $w_{2}$ as a super-solution on $\left[0, \frac{1}{4}\right]$ of

$$
\left.\begin{array}{l}
-u^{\prime \prime}(x) \in f(u(x)), \\
u(0)=u\left(\frac{1}{4}\right)=0 .
\end{array}\right\}
$$

We then use the oddness of $f$ to generate a new solution of $(3.1)$ on $[0,1]$.

It is clear that, by continuing in this way, the result may be established by induction.

The proof when (i), (ii) and (iii)(b) hold is similar.

Proof of Corollary. This follows from the theorem once it is shown that the sub- 
and super- solutions $\phi$ and $\psi$ exist. To see this let $\alpha, \Gamma$ be such that $f(t) \leqslant\left(\pi^{2}-\alpha\right) t+\Gamma$ for all $t \geqslant 0$, and let $\psi$ be the solution of the problem:

$$
\begin{gathered}
-u^{\prime \prime}(x)=\left(\pi^{2}-\alpha\right) u(x)+\Gamma \quad \text { for } x \in(0,1), \\
u(0)=u(1)=0 .
\end{gathered}
$$

Then $\psi>0$ on $(0,1)$ and $\psi$ has property $(*)$. Put $\phi=-\psi$.

\section{References}

1. D. Gilbarg and N. S. Trudinger, Elliptic partial differential equations of second order (Springer, Berlin, 1977).

2. C. A. Stuart, "Maximal and minimal solutions of elliptic differential equations with discontinuous nonlinearities", Math. Z., 163 (1978), 239-249.

3. C. A. Stuart, and J. F. Toland, "A variational method for boundary value problems with discontinuous nonlinearities", J. London Math. Soc. (2), 21 (1980), 319-328.

4. C. A. Stuart, "Elliptic boundary value problems with discontinuous non-linearities", to appear.

Département de Mathématique,

École Polytechnique Fédérale de Lausanne, 6, av. de Cour,

Lausanne CH-1007, Switzerland.
Department of Mathematics, University College,

Gower Street, London WC1E 6BT. 